\title{
Real-time Two-Dimensional Temperature Imaging Using Ultrasound
}

\author{
Dalong Liu and Emad S. Ebbini
}

\begin{abstract}
We present a system for real-time 2D imaging of temperature change in tissue media using pulse-echo ultrasound. The frontend of the system is a SonixRP ultrasound scanner with a research interface giving us the capability of controlling the beam sequence and accessing radio frequency (RF) data in real-time. The beamformed RF data is streamlined to the backend of the system, where the data is processed using a two-dimensional temperature estimation algorithm running in the graphics processing unit (GPU). The estimated temperature is displayed in real-time providing feedback that can be used for real-time control of the heating source. Currently we have verified our system with elastography tissue mimicking phantom and in vitro porcine heart tissue, excellent repeatability and sensitivity were demonstrated.
\end{abstract}

\section{INTRODUCTION}

Noninvasive temperature estimation continues to attract attention as a means of monitoring and guidance for minimally-invasive thermotherapy. Currently, minimallyinvasive RF ablation is the most commonly used form of thermal therapy, but other techniques are being used or investigated. For example, high-intensity focused ultrasound (HIFU) is being evaluated clinically as a form of noninvasive thermal therapy. Successful implementation of real-time noninvasive temperature estimation will be a boon for thermal therapy as it becomes less invasive and as the heating sources become more sophisticated.

Currently, magnetic resonance imaging (MRI) and ultrasound have both been proven to have the temperature sensitivity and spatial resolution necessary to provide noninvasive temperature feedback. The main limitation for MRI is the cost and the potential complication of the heating protocol as the heating equipment must be MR compatible. Ultrasound is relatively less expensive, portable, and can be used in conjunction with almost any heating protocol without adding any significant constraints. Therefore, noninvasive temperature estimation based on ultrasound echo data continues to be an important problem in the area of image-guided minimallyinvasive thermal therapy.

Several methods have been proposed for temperature imaging using pulse-echo ultrasound, but echo shift estimation (using speckle tracking [1]-[3]) and spectral shift estimation (using high resolution spectral estimation [4]) are most commonly used methods. The principle of measurement was validated experimentally by several groups in tissue and tissue-mimicking samples [5]-[7]. Both spectral

Dalong Liu is with the Department of Biomedical Engineering, University of Minnesota, Minneapolis, MN 55455, USA liuxx293@umn . edu

Emad S. Ebbini is with the Department of Electrical Engineering, University of Minnesota, Minneapolis, MN 55455, USA emadeece.umn.edu and echo shift models for estimation of temperature change employed a linear model with a proportionality "constant" related to the temperature coefficient of the speed of sound, $\beta=(\partial c / \partial T) / c$, and the coefficient of thermal expansion, $\alpha$ [8]. These methods, however, currently suffer from some limitations that have hindered their adoption in the clinic. These limitations stem from native tissue deformations (e.g. due to breathing and cardiac cycle) leading to echo and spectral shifts easily masking the (typically very small) temperatureinduced shifts. Heterogeneities in the target tissue as well as the scattering models could result in misinterpretation of the temperature-induced shifts. Tissue strains due to fast switching of the heating source (as in pulsed HIFU or on/off control of RF ablation) also produce artifacts due to undersampling of the tissue displacement fields at typical frame rates of $2 \mathrm{D}$ ultrasound imaging.

In this paper, we present preliminary data to demonstrate a new temperature imaging mode employing high frame rate 2D pulse-echo ultrasound. A number of scan lines from the region of interest (ROI) are interrogated at high frame rate to capture the full thermal and mechanical transients of the HIFU source. This M2D-mode is also suitable for capturing tissue motion and deformation within the ROI due to respiratory and cardiac cycles. We show temperature imaging results from heating tissue-mimicking phantoms as well as in vitro porcine heart. The results clearly demonstrate the advantages of ultrasound as a temperature imaging modality. Specifically, its high sensitivity to small $\left(\mathcal{O}\left(1^{\circ} \mathrm{C}\right)\right)$ temperature change, high resolution (matching that of the imaging probe, and high temporal resolution (500 fps shown in this paper). In addition, the results demonstrate the high repeatability of the measurement.

\section{METHODS}

\section{A. Temperature Estimation}

The temperature change estimation method used in the current system is based on the thermal dependence of the ultrasound echo that accounts for two different physical phenomena: local change in the speed of sound and thermal expansion of the propagating medium due to changes in temperature. The speed of sound $c$ is a function of temperature. In most tissue media around body temperature, $c$ increases with temperature in the range (see [9]). In fatty tissues, $c$ decreases with increasing temperature [10].

In [1], we have described a temperature estimation algorithm based on speckle tracking relating changes in echo location to changes of tissue temperature. This approach was extended and fully described in [3]. It has been shown in [3] 
that

$$
\Delta T(z)=\frac{c\left(T_{0}\right)}{2}\left[\frac{1}{\beta-\alpha}\right] \frac{\partial}{\partial z}(\delta t(z))
$$

where $\alpha=(\partial d(T) / \partial T) / d(T)$ is the linear coefficient of thermal expansion and $\beta=(\partial c(T) / \partial T) / c(T)$ is the thermal coefficient of the speed of sound,for a homogeneous medium these parameters can be determined experimentally. Therefore, (1) suggests that temperature-change estimates can be obtained by first tracking the cumulative echo timeshift at each location, and then differentiating along the axial direction $z$ and filtering along both axial and lateral $x$ directions. This method was explained and discussed in detail in [3].

\section{B. System Architecture}

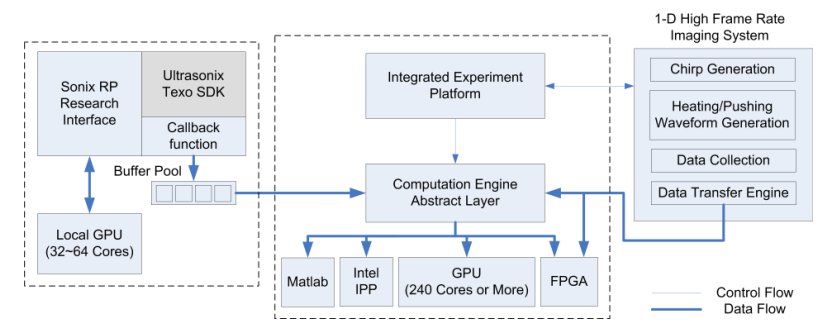

Fig. 1. A block diagram of the imaging system as it is currently implemented

The system shown in Figure 1 is used for applying pulsed HIFU, monitoring tissue response and real-time processing of acquired data.

A Virtex2Pro (Digilent Inc, WA) FPGA board is dedicated for HIFU source and synchronized frame trigger generation. It can work either in open-loop mode where a pre-defined sequence get executed or close-loop mode where each segment of the waveform (usually in tens of ms) is updated by the host system, in which case the estimated temperature can be used as feedback input to modulate the HIFU output. A SonixRP (Ultrasonix, Canada) ultrasound scanner loaded with custom designed program is used for pulse-echo data collection, collected data is then streamlined to another PC through Gigabit Ethernet for realtime data processing. Equipped with a many-core GTX285 (nVIDIA, Santa Clara, CA) GPU, the data processing computer can easily handle the intensive compuation required by high resolution (both spatial and temporal) speckle tracking and separable 2D post filtering. Note that the same computer system is also used for HIFU source generation by controlling the FPGA board, the ability to communicate between the GPU and FPGA in realtime also allows the real-time feedback control of heating source.

The ultrasound scanner works primarily in two modes: the $B$ mode for image guidance and another mode that we call it M-2D, in this mode only part of the transducer (region of interest) is active so that a much higher temporal sampling rate can be achieved (e.g., 10 active Alines with a frame rate of $500 \mathrm{~Hz})$.

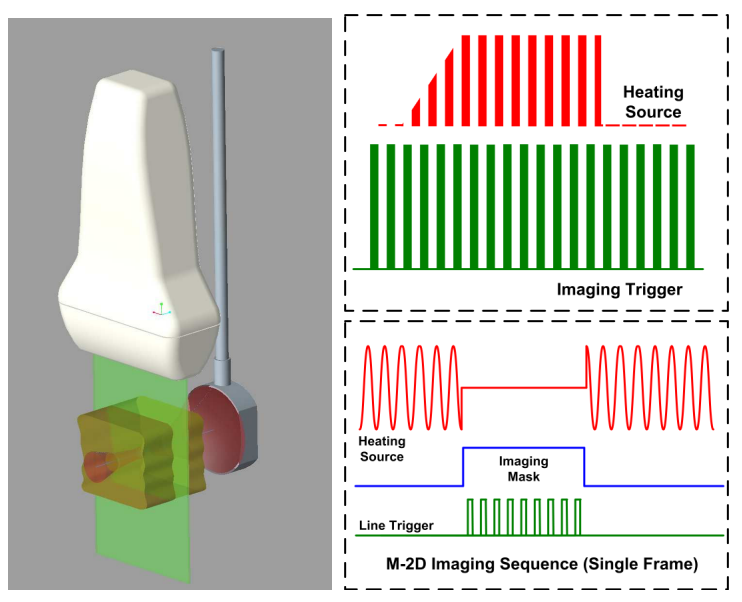

Fig. 2. An experimental setup used in imaging localized temperature fields generated using a focused therapy transducer used in noninvasive surgery. Note the imaging and therapy pulses are completely synchronized using the FPGA platform shown in Figure 1.

\section{Real-time Data Processing}

Temperature imaging relies on accurate estimates of incremental time-shifts which are much smaller than RFecho sampling period, the method we used is based on 2-D complex correlation of two subsequent frames of RF echo [3]. Despite being more computationally efficient than interpolation based method, speckle tracking based on the phase of the complex cross correlation is still beyond the reach of realtime implementation on CPU-based system. The realtime data processing engine we developed is based on a GTX285 GPU (240 stream processors at $1.47 \mathrm{GHz}$ ), to fully utilize the massive computational power offered by the hardware, we re-designed the algorithm to take full advantage of this highly parallel architecture, Figure 3 shows the diagram for the GPU implementation, note that for each processing stage, fine-grained partition is performed for the algorithm in a data independant manner so that all 240 processors are working efficiently on individual block of data. As an emerging technology for high performance computing, GPU parallel processing has several advantages of over the CPU counterpart:

1) Many-core versus multi-core. A high-end GPU have hundreds of cores (e.g., 480 cores in GTX295) optimized for arithmetic operations where CPU typically only have 4 or 8 cores, so GPU is more suitable for computation intensive task.

2) Minimum task creation and zero task switching overhead make GPU more suitable for fine-grained parallel processing.

3) Intuitive parallel programming model yields a straightfoward and efficient implementation

One thing we'd like to mention is that current implementation only takes less than $1 / 10$ of the total computational power this single GPU can offer (our system does allow expansion of up to 3 GPUs with equal or more processing power), so we really have a lot of room to incorporate more advanced algorithms to address various issues with temperature esti- 
mation and still run everything in real-time.

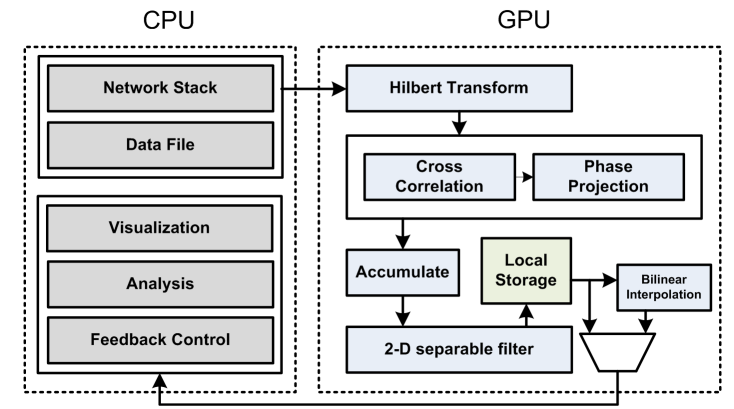

Fig. 3. A block diagram of GPU implementation

\section{Experiment Materials}

Experiments were performed on elastography phantoms fabricated from gelatin, graphite, water and glutaraldehyde in quantities suggested in [11]. The thermal properties are measured by slowly heating and cooling of the gel phantom in a water bath, as suggested in [3].

In vitro experiment was performed on fresh store-bought porcine heart tissue.

\section{E. Experiment Procedure}

All heating experiments were done with a $4 \mathrm{MHz} \mathrm{HIFU}$ transducer (Focus Surgery, Inc., IN) and monitored by the SonixRP scanner. A linear array probe (LA14-5/38, 1 cycle transmit pulse, centered at $9.5 \mathrm{MHz}$ for phantom and $6 \mathrm{MHz}$ for heart tissue) was used for data collection at a frame rate of $500 \mathrm{fps}$ with limited imaging range and limited number of scan lines.

The heating/data-acquisition sequence is illustrated in Figure 2:

1) It starts with $400 \mathrm{~ms}$ tracking without application of heating beam to serve as baseline temperature.

2) The heating beam starts to ramp up to full intensity in $200 \mathrm{~ms}$ and stays on for another $1.5 \mathrm{~s}$.

3) Another $2 s$ of tracking data is collected after the heating beam is turned off.

Note that the tracking and heating are interleaved and strictly synchronized to eliminate the interferences from the heating beam to tracking sequence.

\section{RESULTS}

\section{A. System Validation with Elastography Phantom}

To test system repeatibility, we set up experiments that heat and monitor the same location in phantom for multiple times. Figure 4 shows one data set that we repeated the experiments for 8 times with 30 seconds interval between each experiment to allow the phantom to cool down. The mean value of peak temperature is $2.62^{\circ} \mathrm{C}$, while the standard deviation is $0.04^{\circ} \mathrm{C}$, which shows excellent repeatability for the system.

In another experiment, we used exactly the same heating parameters at different target locations and monitored the

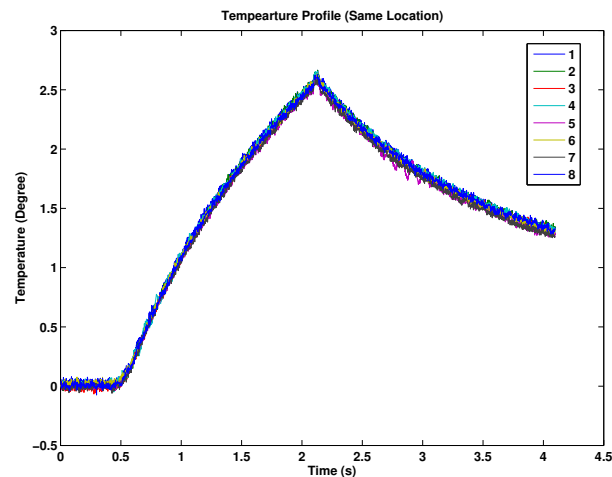

Fig. 4. Temperature estimations for 8 heating experiments. All experiments were done at the same location

temperature changes before, during and after the application of the heating pulse. Figure 5 shows a data set from 5 heating locations. Larger variations (standard deviation of $0.14^{\circ} \mathrm{C}$ ) were observed compared to the previous case, which is most probably due to the slight differences in thermal properties and geometry of the phantom we used.

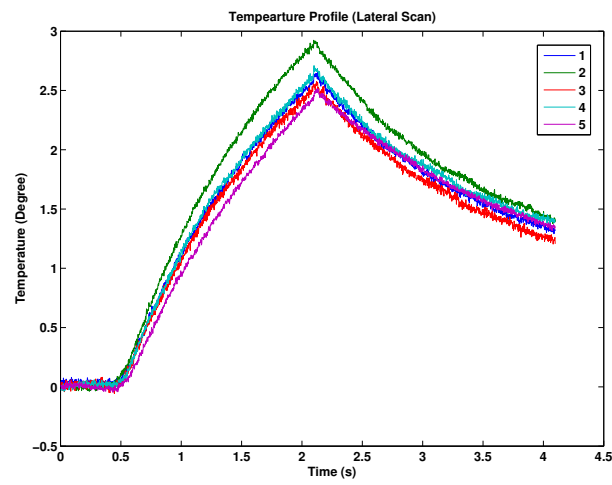

Fig. 5. Temperature estimations for 5 heating experiments. Experiments were performed at different locations at same depth

\section{B. In Vitro Porcine Heart Tissue Experiment}

Estimated temperature data resulting from heating the in vitro heart tissue is shown in Figure 7. Since the data is 3D (2D+time), there are many ways of visualizing the data. We choose to display three projections based on a given coordinate $(\mathrm{X}, \mathrm{Z}, \mathrm{T})$, where $\mathrm{X}$ stands for lateral position, $\mathrm{Z}$ is the axial position and $\mathrm{T}$ is in the time direction. Projections shown in Figure 7 is taken near the peak temperature in 3D data set.

Figure 8 shows the overlay of temperature profile across the focal spot from 9 experiments. Result shows reasonable repeatability from the in vitro data set. In this case, mean value of $1.65^{\circ} \mathrm{C}$ and standard deviation of $0.1{ }^{\circ} \mathrm{C}$ were observed.

\section{CONCLUSIONS AND FUTURE WORKS}

The system we presented here provides a complete solution including HIFU source generation, high frame rate 


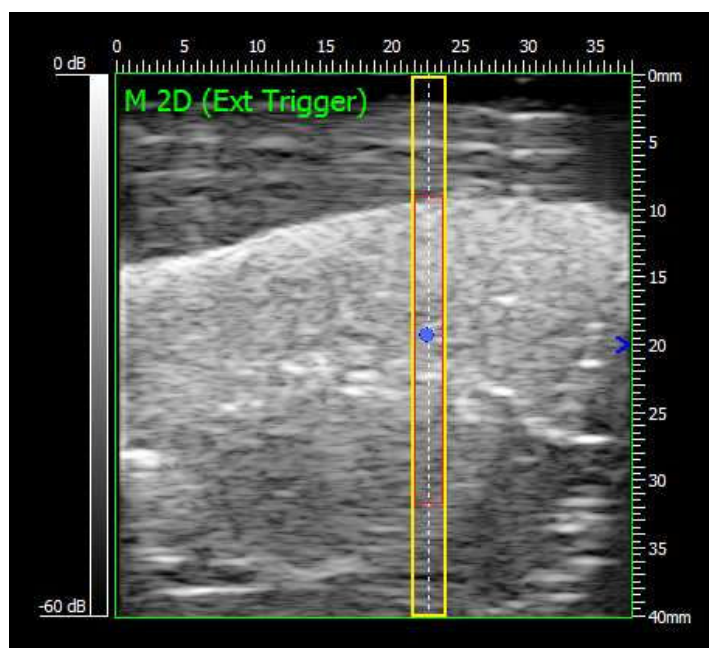

Fig. 6. Guidance image for heating experiment. Yellow box shows the region where $\mathrm{M}-2 \mathrm{D}$ mode data were collected and processed, red box shows the data actually displayed in Figure 7, blue dot is the desired heating spot

data capture and true real-time data processing. Currently we are working on the feedback control feature which regulates the HIFU source by incorporating the real-time estimated temperature to maintain a set temperature in the heated region. Note that there are limitations with the model in Section II-A, such as the thermoacoustic lens effect. Difficulties at higher temperatures and fat content dependency of temperature coefficient. various solutions had been proposed by us and other researchers to address these issues. With the computational power and flexibility from current system, we are quite confident that these solutions can be applied to our system as well. This system would provide the key component in a complete image guidance modality for thermal therapy.

\section{ACKNOWLEDGMENTS}

This work is funded in part by Grant EB006893 from the National Institutes of Health.

\section{REFERENCES}

[1] R. Seip, P. VanBaren, C. Simon, and E. Ebbini, "Non-invasive spatiotemporal temperature change estimation using diagnostic ultrasound," 1995, pp. 1613-1616.

[2] R. Maass-Moreno and C.A. Damianou, "Noninvasive temperature estimation in tissue via ultrasound echo shifts. part i. theoretical model," The Journal of the Acoustical Society of America, vol. 100, pp. 2514-2521, 1996.

[3] C. Simon, P. VanBaren, and E.S. Ebbini, "Two-dimensional temperature estimation using diagnostic ultrasound," IEEE Trans. Ultrason., Ferroelect., Freq. Contr., vol. 45, pp. 989-1000, July 1998.

[4] R. Seip and E. Ebbini, "Non-invasive estimation of tissue temperature response to heating fields using diagnostic ultrasound," IEEE Trans. Biomed. Eng., vol. 42, no. 8, pp. 828-839, 1995.

[5] Naomi R Miller, Jeffrey C Bamber, and Paul M Meany, "Fundamental limitations of noninvasive temperature imaging by means of ultrasound echo strain estimation," Ultrasound in Medicine and Biology, vol. 28, pp. 1319-1333, 2002.

[6] M. Pernot, M. Tanter, J. Bercoff, K. Waters, and M. Fink, "Temperature estimation using ultrasonic spatial compounding," IEEE Trans. Ultrason., Ferroelect., Freq. Contr., vol. 51, no. 5, pp. 606-615, 2004.

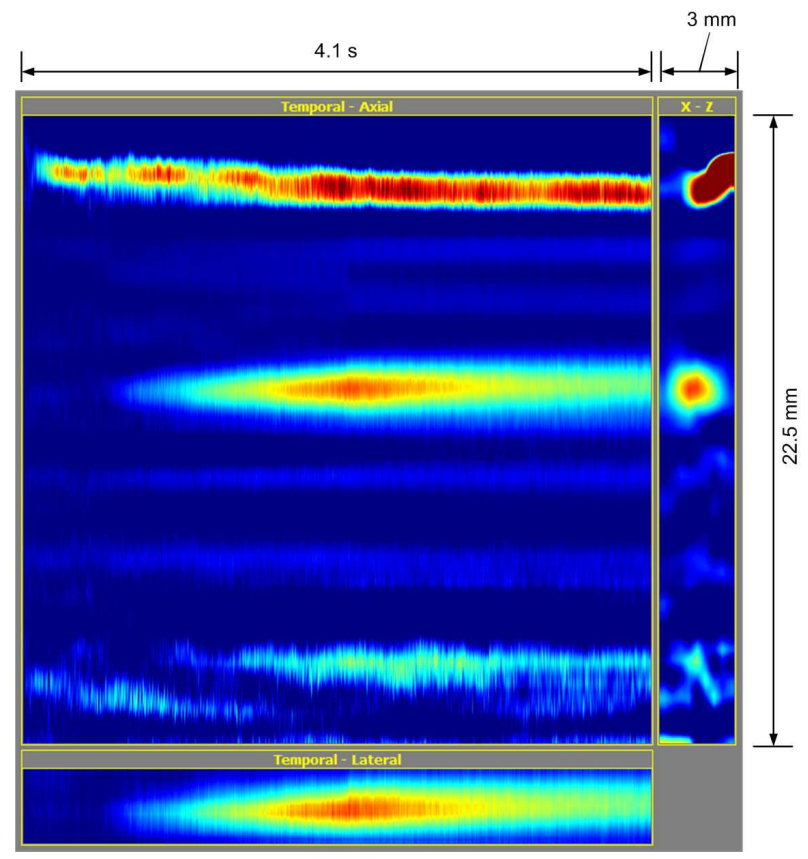

Fig. 7. Example visualization of the $3 \mathrm{D}(2 \mathrm{D}+\mathrm{time})$ temperature data. The data set is 10-scan lines wide, 4.1-second duration at $500 \mathrm{fps}$. The focus of the therapeutic transducer is orthogonal to the imaging plane. $\mathrm{X}-\mathrm{Z}$ stands for Lateral - Axial.

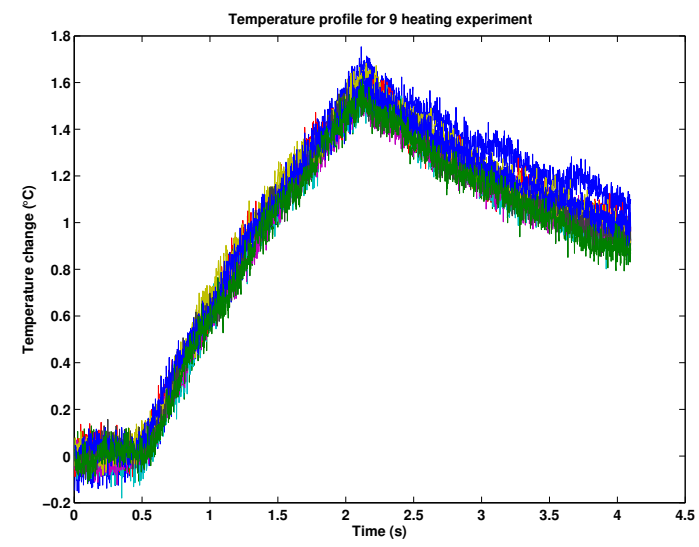

Fig. 8. Overlay of temperature profile across the focal spot from 9 experiments at the same location

[7] Remi Souchon, Guillaume Bouchoux, Eva Maciejko, Cyril Lafon, Dominique Cathignol, Michel Bertrand, and Jean-Yves Chapelon, "Monitoring the formation of thermal lesions with heat-induced echostrain imaging: A feasibility study," Ultrasound in Medicineand Biology, vol. 31, pp. 251-259, 2005.

[8] A. Nasiri Amini, E.S. Ebbini, , and T. Georgiou, "Noninvasive estimation of tissue temperature via high-resolution spectral analysis techniques," IEEE Trans. Biomedical Engineering, vol. 52, no. 2, pp. 221-228, 2005.

[9] L.E. Kinsler and A. R. Frey, Fundamentals of Acoustics, Wiley, New York, NY, 3rd edition, 1982.

[10] S. A. Johnson J. F. Greenleaf, P. J. Thomas and R. C. Bahn, "ultrasonic tissue characterization II," pp. 227-233, 1979.

[11] K. R. Nightingale, M. L. Palmeri, R. W. Nightingale, and G. E. Trahey, "On the feasibility of remote palpation using acoustic radiation force," J. Acoust. Soc. Am., vol. 110, pp. 625-634, July 2001. 